\title{
Transport fuel demand responses to fuel price and income projections: Comparison of integrated assessment models
}

\author{
O.Y. Edelenbosch a,b,*, D.P. van Vuuren ${ }^{\mathrm{a}, \mathrm{b}}$, C. Bertram ${ }^{\mathrm{c}}$, S. Carrara ${ }^{\mathrm{d}}$, J. Emmerling ${ }^{\mathrm{d}}$, H. Daly ${ }^{\mathrm{e}}$, \\ A. Kitous ${ }^{\mathrm{f}}$, D.L. McCollum ${ }^{\mathrm{g}}$, N. Saadi Failali ${ }^{\mathrm{e}}$ \\ a PBL Netherlands Environmental Assessment Agency, Bezuidenhoutseweg 30, 2594 AV Den Haag, The Netherlands \\ ${ }^{\mathrm{b}}$ Copernicus Institute of Sustainable Development, Utrecht University, Heidelberglaan 2, 3584 CS Utrecht, The Netherlands \\ ${ }^{\mathrm{c}}$ Potsdam Institute for Climate Impact Research, Telegrafenberg, 14473 Potsdam, Germany \\ ${ }^{\mathrm{d}}$ Fondazione Eni Enrico Mattei (FEEM) and Centro Euro-Mediterraneo sui Cambiamenti Climatici (CMCC), Corso Magenta 63, 20123 Milan, Italy \\ ${ }^{\mathrm{e}}$ UCL Energy Institute, University College London, Upper Woburn Place, London WC1H 0NN, United Kingdom \\ ${ }^{\mathrm{f}}$ European Commission, JRC IPTS, Edificio Expo; C/Inca Garcilaso, 3, E-41092 Sevilla, Spain ${ }^{1}$ \\ ${ }^{\mathrm{g}}$ International Institute for Applied Systems Analysis (IIASA), Schlossplatz 1, 2361 Laxenburg, Austria
}

\section{A R T I C L E I N F O}

\section{Article history:}

Received 6 July 2016

Revised 21 January 2017

Accepted 5 March 2017

Available online 28 March 2017

\section{Keywords:}

Transportation

Energy modelling

Model evaluation

Price elasticity

Income elasticity

\begin{abstract}
A B S T R A C T
Income and fuel price pathways are key determinants in projections of the energy system in integrated assessment models. In recent years, more details have been added to the transport sector representation in these models. To better understand the model dynamics, this manuscript analyses transport fuel demand elasticities to projected income and fuel price levels. Fuel price shocks were simulated under various scenario assumptions to isolate price effects on energy demand and create a transparent environment to compare fuel demand response. Interestingly, the models show very comparable oil price elasticity values for the projected first 10-20 years that are also close to the range described in the empirical literature. When looking at the very long term (30-40 years), demand elasticity values widely vary between models, between 0.4 and -1.9 , showing either continuous demand or increased demand responses over time. The latter can be the result of long response time to fuel price shocks, availability of new technologies, and feedback effects on fuel prices. The projected transport service demand is more responsive to changes in income than fuel price pathways, corresponding with the literature. Calculating the models' inherent elasticities proved to be a suitable method to evaluate model behaviour and its application is also recommended for other models as well as other sectors represented in integrated assessment models.
\end{abstract}

(c) 2017 Elsevier Ltd. All rights reserved.

\section{Introduction}

Integrated Assessment Models (IAMs) have been developed to model the evolution of the global energy and land-use systems for the coming century. They have extensively been used to project greenhouse gas emissions and to identify costeffective mitigation strategies (Luderer et al., 2012; Clarke et al., 2009). In the past, IAMs tended to represent energy demand

\footnotetext{
* Corresponding author.

E-mail addresses: Oreane.Edelenbosch@pbl.nl (O.Y. Edelenbosch), Detlef.vanvuuren@pbl.nl (D.P. van Vuuren).

1 Disclaimer: The views expressed are purely those of the writer and may not in any circumstances be regarded as stating an official position of the European Commission.
} 
sectors in a rather stylised manner, while presenting energy supply in more detail. Energy demand sectors are complex, both in terms of the many sub-sectors with numerous technologies and in the heterogeneity of consumers that use the services requiring energy. These sectors are therefore more difficult to represent in quantitative models.

Energy demand reduction can, however, have important contribution to emission reduction (Luderer et al., 2012; Sugiyama et al., 2014; Riahi et al., 2012). In recent years, more details of the energy demand side have been incorporated in IAMs, in order to better understand demand dynamics and the role of efficiency in mitigation strategies. This is especially the case for the transport sector, where infrastructure, behaviour and technology considerations have been addressed, as described in several articles in the Transport Research Part D special issue on transport modelling in IAMs (McCollum et al., 2017; Ó Broin and Guivarch, 2017; Mittalet al., 2017; Karkatsoulis et al., 2017; Carrara and Longden, 2017).

The models have various representations of the transport system, some with more technology detail, and others providing a more aggregated demand formulation. Several studies compare IAM transport sector outcomes (Girod et al., 2013; Edelenbosch et al., 2017; Pietzcker et al., 2014), and show a variation in projected growth of transport service demand, fuel switching and efficiency change (Edelenbosch et al., 2017). Intermodal comparison studies are informative, as they provide a range of plausible pathways. However, as the models have become more complex, it becomes less easy to understand why model results differ (Sugiyama et al., 2014). Kriegler et al. (2015) indicate that, besides intermodal comparisons, diagnostic analysis which characterise model dynamics, are very relevant to explain model differences. This type of analysis is not aimed to explore realistic policy scenarios, but to identify typical model responses to a single policy signal. So far, a detailed diagnostic analysis of transport model responses to key drivers in IAMs has not been performed.

Income and fuel price levels are key model drivers. Income relates to the money available to spend on transport activities and fuel price affects the benefits of energy efficiency of technologies used and of switching to alternative fuels. Moreover, the implementation of a carbon tax, which is the commonly used mitigation policy instrument in IAMs, will impact fuel prices. Elasticities of transport fuel demand are used as measure for how sensitive demand is to changes in -in this caseeither income or prices. In this study, the transport models' implicit fuel demand elasticities are explored, by comparing demand responses to various fuel price and income trajectories. The aim is twofold; first to better understand model dynamics through a diagnostic experiment, and second comparing the model dynamics to empirical data as a validation test. A large number of empirical studies have analysed the sensitivity of transport demand to changes in fuel price and income (Litman, 2013; McCollom and Pratt, 2004), expressed in elasticities, to inform transport planners and policymakers (Small and Van Dender, 2007). Moreover, quantifying the model response to elasticity values provides the opportunity to translate model dynamics of models that consider details of transport modes and technologies into relatively aggregate models. A comparable exercise has been performed for demand models by Hogan and Sweeney (Hogan and Sweeney, 1981). They conclude that the implicit elasticity calculation method is appropriate for comparing demand model dynamics, and they recommend modellers to make this a standard component of their documentation to better understand the model dynamics.

An overview of the various models and methods used to calculate elasticities and scenarios that were run by the models are discussed in Section 2. The models' transport consumption response to varying fuel prices and income scenarios compared to the empirical data is presented and discussed in Section 3. Underlying changes, such as efficiency effects and changes in the kilometres travelled, are addressed separately. Section 4 provides tentative conclusions about the variations between models and discusses the implications of the projections of energy transitions and the role of climate policy.

\section{Method}

With everything else remaining constant, fuel demand elasticities measure the percentage change in demand due to a $1 \%$ increase in price or income. A set of scenarios was designed to estimate price and income elasticities for transport demand in six global integrated assessment models. Elasticities of fuel consumption, but also, for those models in this study containing sufficient detail, service demand and efficiency responses for specific transport modes. This section provides an overview of the models, the fuel and income scenarios, and the elasticity calculation method.

\subsection{Models and baseline scenario}

The IAMs included in this study are IMAGE, MESSAGE, POLES, REMIND, TIAM-UCL and WITCH. ${ }^{2}$ These form a set of wellknown IAM models that contributed to key assessments and also cover a wide range of different methods (see Table 1 and Supplementary Information).

Of the six IAMs, POLES, IMAGE and TIAM-UCL have a more technology-rich representation of transport demand. The projected kilometres travelled, which are related to population and GDP, are distributed over the transport modes either based on exogenous assumptions (TIAM-UCL and POLES) or endogenously on their price and speed (IMAGE). Per transport mode, different technologies are considered that compete on the basis of exogenous technology cost and endogenous fuel cost. POLES and IMAGE are both recursive dynamic simulation models and TIAM-UCL is a linear optimisation model.

\footnotetext{
${ }^{2}$ The MESSAGE transport module used in this study is a simpler version than the one used in other papers (e.g.. McCollum et al., 2017) of the special issue "Transport in IAMs", that this paper is part of. Other models employed might also not exactly match those versions employed in other papers of the special issue.
} 
Table 1

Overview of key characteristics of the transport models.

\begin{tabular}{|c|c|c|c|c|}
\hline Name & $\begin{array}{l}\text { Model } \\
\text { type }\end{array}$ & Solution methods & Service demand driver per transport mode & $\begin{array}{l}\text { End use technology } \\
\text { representation }\end{array}$ \\
\hline IMAGE & $\mathrm{PE}$ & Recursive dynamic simulation & $\begin{array}{l}\text { GDP, population, fuel price, travel time, mode } \\
\text { characteristics }\end{array}$ & All modes \\
\hline POLES & PE & Recursive dynamic simulation & GDP/income, population, fuel prices & All modes \\
\hline MESSAGE & GE & Intertemporal optimisation & GDP, population, fuel price & $\begin{array}{l}\text { All modes (aggregated } \\
\text { together) }\end{array}$ \\
\hline REMIND & GE & $\begin{array}{l}\text { Intertemporal optimisation with } \\
\text { perfect foresight }\end{array}$ & $\begin{array}{l}\text { GDP growth, fuel prices, elasticity of substitution in } \\
\text { CES function }\end{array}$ & LDV \\
\hline $\begin{array}{l}\text { TIAM- } \\
\text { UCL }\end{array}$ & PE & Intertemporal optimisation & Linear relation to GDP and population & All modes \\
\hline WITCH & GE & $\begin{array}{l}\text { Intertemporal optimisation with } \\
\text { perfect foresight }\end{array}$ & $\begin{array}{l}\text { GDP, population, elasticity of substitution in CES } \\
\text { function }\end{array}$ & LDV and road freight \\
\hline
\end{tabular}

In REMIND, the mobility demand for all modes of transport are input to a nested CES production function that ultimately produces GDP. REMIND differentiates between four other transport modes besides light duty vehicles (LDVs). The representation of transport in the version of MESSAGE used in this study, captures only fuel switching and price-induced demand responses (McCollum et al., 2014). Importantly, the entire sector is modelled as one; all motorised transport modes are aggregated together into a single demand category.

Finally, in WITCH, road transport kilometre demand (LDVs and freight) is derived based on GDP and population growth. This demand can be met by different vehicles (traditional, hybrid, plug-in hybrid, battery electric vehicles) and fuel types, which compete based on cost. The investment costs of batteries endogenously decrease, following a global learning rate via dedicated $R \& D$ investments. The remaining part of the transport sector is modelled in a top-down fashion and included in the aggregated non-electric vehicle sector in the CES structure.

\subsection{Fuel price elasticity scenarios}

In line with previous studies that tested demand elasticities inherent in models, scenarios with fuel prices shocks are compared to the original price pathway in the models' baseline scenario (Hogan and Sweeney, 1981; Jaccard and BeylinStern, 2014). The shocks are applied to (1) oil \& natural gas, (2) biofuels and (3) electricity from 2020 to 2070 , changing the price with respect to a reference price trajectory, by $-50 \%,+50 \%$ and $+100 \%$ (see Table 2 ). Based on experience with model demand responses to carbon prices the expectation was that fuel price shocks of 50-100\% would be needed before there would be a significant demand response. The fuel price is increased at the final energy level for all demand sectors; however, the focus of our analysis is only on the transport demand response.

Fig. 1 shows the baseline transport oil price used in each model (at the end-use level; global average) (panel a), as well as the fuel price change, relative to the baseline, in the $-50 \%$ scenario and $+100 \%$ scenario (panel b). The WITCH and TIAM-UCL scenarios do not include end-use taxes in their prices, therefore these models use a lower price pathway. All models project oil prices to increase as a result of resource depletion in the baseline, but the extent of this effect varies. The variations in oil price development between models ultimately result in different fuel shocks in absolute terms. Price jumps are implemented as exogenous shocks. In two models (IMAGE, POLES), this is implemented by replacing the endogenous prices by an exogenous input. In the other models (TIAM-UCL, MESSAGE, REMIND and WITCH), where this would interfere with the model solution, price increases/decreases were added to the endogenously calculated final energy prices - thus mimicking additional taxes or subsidies. Here, dynamic model responses and feedback effects can clearly be observed.

In some models, due to higher fuel prices, the demand for a particular fuel (and its primary resource, e.g., crude oil) is reduced. As a result however, in those models where the fuel prices are not an exogenous input, final energy prices tend to move away from the price shock pathway towards the original price pathway, in the long run. This effect is the largest in MESSAGE, but can also be seen in the TIAM-UCL and REMIND projections. In REMIND, the perfect foresight feature leads to a reduction in the price change effect already by 2020 (when the shock is introduced). The reduction in demand due to the exogenous price increase, in this case, has led to a relaxation of the scarcity and thus to a reduction in the endogenous price component. Yet, despite the variance in fuel price pathways, since price demand elasticities are calculated relative to price changes (and not to price levels), even with smaller price changes it is still possible to compare elasticities between models.

\subsection{Income elasticity scenarios}

Two extra scenarios with different income pathways are run to analyse income elasticities (see Table 2, Scenarios 11 and 12 and Fig. 2). In the baseline scenario, the models have implemented the Shared Socio-Economic Pathway (SSP) 2 assumptions on GDP and population growth. The SSPs are a scenario framework that defines pathways of the evolution of society and ecosystems in the next century. Within this framework SSP2 is the middle of the road scenario. The alternative GDP 
Table 2

Scenario design to calculate price demand and income demand elasticities. Descriptions of Scenarios 2 to 10 indicate the price jumps relative to the baseline scenario, for the three fuel types considered. Ref indicates the unaltered reference fuel price trajectory in the baseline of each model. Scenario descriptions of Scenarios 11 and 12 indicate the varying income pathways.

\begin{tabular}{|c|c|c|c|c|}
\hline \multirow[t]{2}{*}{ Scenario } & & \multicolumn{3}{|c|}{ Price change per fuel type } \\
\hline & & Oil \& Natural gas & Electricity & Biofuel \\
\hline 1 & & Ref & Ref & Ref \\
\hline 2 & & $-50 \%$ & Ref & Ref \\
\hline 3 & & Ref & $-50 \%$ & Ref \\
\hline 4 & & Ref & Ref & $-50 \%$ \\
\hline 5 & & $+50 \%$ & Ref & Ref \\
\hline 6 & & Ref & $+50 \%$ & Ref \\
\hline 7 & & Ref & Ref & $+50 \%$ \\
\hline 8 & & $+100 \%$ & Ref & Ref \\
\hline 9 & & Ref & $+100 \%$ & Ref \\
\hline 10 & & Ref & Ref & $+100 \%$ \\
\hline Scenario & \multicolumn{4}{|l|}{ Income change } \\
\hline 11 & \multicolumn{4}{|l|}{ SSP1 GDP assumptions } \\
\hline 12 & \multicolumn{4}{|l|}{ SSP3 GDP assumptions } \\
\hline
\end{tabular}
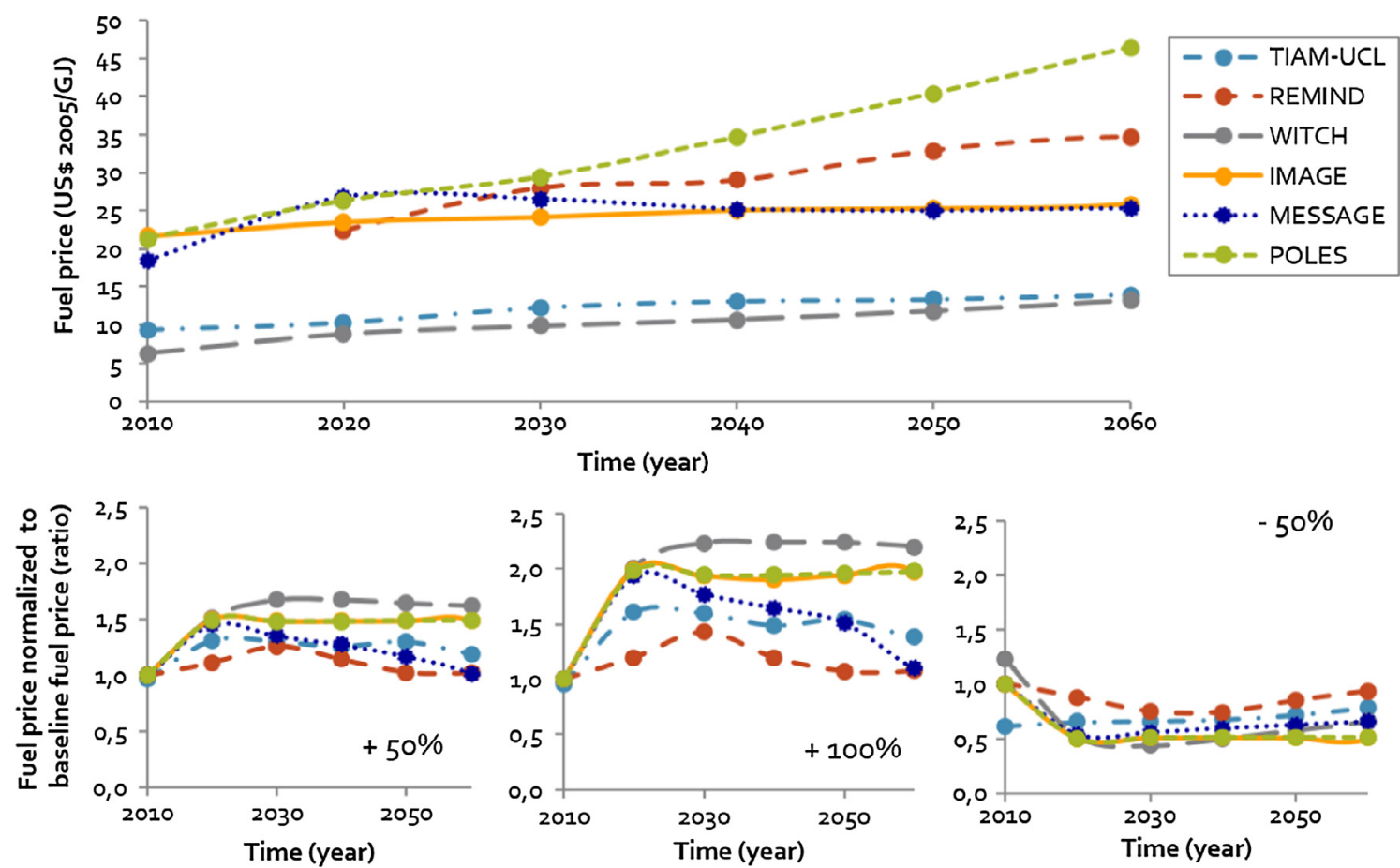

Fig. 1. Global average price of transport oil in the baseline scenario (Scenario 1) (top) and the relative increase in oil price compared to this baseline (bottom), for the price shock scenarios of $+50 \%$ (Scenario 5), $+100 \%$ (Scenario 8 ) and $-50 \%$ (Scenario 2 ). Note that in the figure the average global prices are shown. The moving away effect at the global level can be larger than at the regional level as the average fuel prices also can be affected by regions, with lower or higher than average fuel prices, accumulating a larger share of the global transport final energy use.

pathways are based on SSP1 and SSP3 which assume respectively low and high challenges for mitigation and adaptation. Within that narrative SSP1 follows higher and SSP3 follows lower economic development than SSP2 (O’Neill et al., 2014 ). ${ }^{3}$

\subsection{Price and income elasticity calculation method}

The scenarios described above allow to calculate elasticities. The various fuel quantities and prices can be compared at the same point in time under various scenario assumptions. This allows to compute the elasticities without having to correct for other covariates or confounding factors (which can obviously not be done for empirically derived elasticities). Based on two different scenarios ( 1 and 2), $Q_{i 1}$ and $Q_{i 2}$ denote the quantity consumed, which can be service demand (kilometres travelled),

\footnotetext{
${ }^{3}$ The SSP1, SSP2 and SSP3 GDP pathway assumptions are published on https://secure.iiasa.ac.at/web-apps/ene/SspDb.
} 


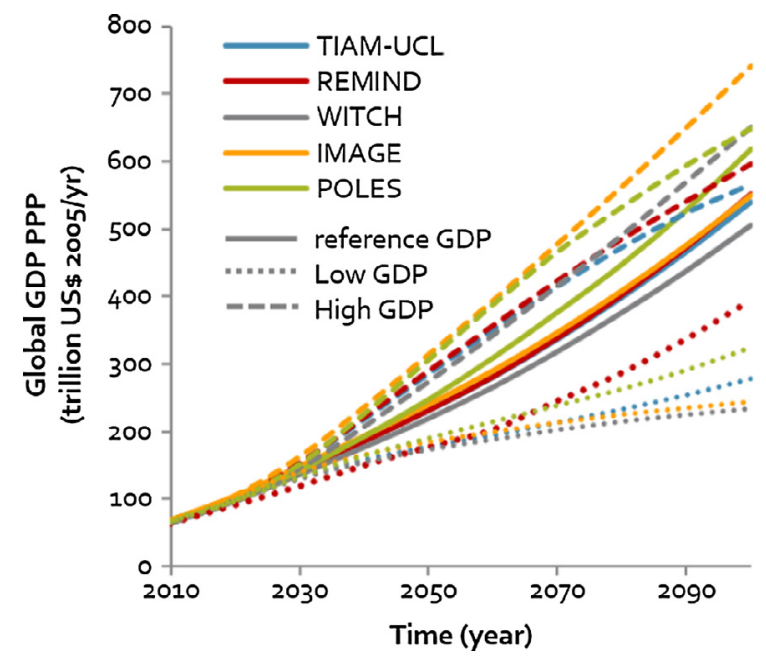

Fig. 2. GDP pathways implemented in the models. The solid line is the SSP2 baseline (Scenario 1), the dashed line is the higher GDP pathway (Scenario 11) and the dotted line represents the lower GDP pathway (Scenario 12).

final energy use, or energy efficiency (energy use per kilometre travelled) of category or fuel $i$. Similarly, $P_{i 1}$ and $P_{i 2}$ denote the price of fuel $i$ in both scenarios. Given these four values, the arc price elasticity can be calculated through a logarithmic function:

$$
\eta_{Q, i}=\frac{\log \mathbf{Q}_{i 2}-\log \mathbf{Q}_{i 1}}{\log \boldsymbol{P}_{i 2}-\log \boldsymbol{P}_{i 1}}
$$

where $\eta_{Q, i}$ measures the price elasticity of quantity $Q$ with respect to the price of fuel $i$.

In this case, various price projections for a given future year are compared, and there is no beginning or end point between those points. The arc elasticity can therefore be approximated by a mid-point formulation on the basis of the average value of the independent variables (Litman, 2013):

$$
\eta_{Q, i}=\left(\frac{\Delta Q_{i}}{0.5\left(Q_{i 1}+Q_{i 2}\right)}\right) /\left(\frac{\Delta P_{i}}{0.5\left(P_{i 1}+P_{i 2}\right)}\right)
$$

where the percentage change between Scenarios 1 and 2 is calculated relative to the average value between the two.

The elasticities are calculated for the years 2030 and 2060. Some models work with 10-year time steps, which would make 2030 the first year for which price change effects can be analysed and 2060 the last. In the literature, there is a differentiation between short-term and long-term elasticities, as the full impact of a price change can take several years to wear out. Short term is often considered less than two years, while long term refers to more than 10 years. It has been found that long-term elasticities are higher (and can be up to three times as high (Dargay and Gately, 1997)) than short-term elasticities. Compared to the literature all those calculated in this study are long-term elasticities, in line with the models' longterm perspective, mostly with an end-of-the-century time horizon. Both the long term (10 years) and the very long term (40 years) are compared to the long-term transport elasticity values described in the empirical literature.

\subsection{Cross-price market share semi-elasticities}

To examine fuel consumption responses to price changes in other fuels, typically standard cross-price elasticities are used (e.g., Ziemba and Schwartz, 2012). However, this approach does not always yield meaningful results: if market shares of alternative fuels are small, such as currently is the case for biofuel and electricity this results in difficult-to-compare high elasticity responses to a slight change in demand. Therefore, market share elasticities are computed (as introduced in Bucklin et al. (1998)). The market shares of different fuels $i$ are defined as:

$$
M S_{i}=\frac{Q_{i}}{\sum_{j=1}^{l} Q_{j}}
$$

Based on these market shares, the changes in absolute values of the market shares $M S_{i}$ are computed for the different fuel types $i$ due to changes in the price of fuel $j$, resulting in cross-price market share semi-elasticities, ${ }^{4}$ which we define as:

\footnotetext{
${ }^{4}$ Note that the same arc elasticity approach is used as before. Moreover, the definition of the semi elasticity here uses the absolute change in a value due to a percentage change in the price.
} 


$$
\eta_{M S i, j} \equiv \frac{M S_{i, 1}-M S_{i, 2}}{\frac{\Delta P_{j}}{0.5\left(P_{j, 1}+P_{j, 2}\right)}}
$$

These market share elasticities can be interpreted as changes in the market share of each fuel i due to a $1 \%$ increase in the price of fuel $\mathbf{j}$ (or by multiplying them by 100 , they represent the (approximate) market share change in percentage points due to a doubling of the price of fuel $\mathrm{j}$ ). These elasticities sum to zero, $\sum_{\mathrm{i}=1}^{\mathrm{l}} \eta_{\mathrm{MSi}, \mathrm{j}}=0$, since market shares always add to one. Therefore, these cross-price elasticities ${ }^{5}$ isolate the fuel switching effect due to price changes as a result of efficiency improvements and demand changes discussed above. ${ }^{6}$

\section{Models' inherent demand elasticity results}

\subsection{Oil and alternative fuel responses}

The absolute change in energy demand, compared to the baseline in 2030 and 2060, of transport oil and alternative fuel $(\mathrm{AF})^{7}$ in response to the oil and natural gas price shocks (Scenarios 2, 5 and 8) are shown in Fig. 3. In 2030 (i.e., 10 years after the applied shock) all models show a decrease in oil demand and an increase in alternative fuel under higher oil and natural gas prices, and vice versa. Most models show a stronger response to the price shocks in 2060 than those in 2030, with higher demand-price slopes (right side vs left side of Fig. 3). POLES is the only model to project the absolute change in oil demand to become less while the fuel price jump becomes larger over time. WITCH shows a relatively mild response to the changing fuel price as well, while IMAGE, REMIND, MESSAGE and TIAM UCL show significant responses. In MESSAGE, oil demand ranges from 35 to $290 \mathrm{EJ} / \mathrm{year}$ in 2060 between the higher and lower price scenario, implying that the transport system has completely changed in response to 40 years of widely diverging price trajectories. As MESSAGE, TIAM-UCL and REMIND show strong feedback effects on the price trajectory, moving back to the original fuel price pathway, here very high price elasticities can be expected.

In all models, the decrease in oil is greater than the increase in alternative fuel demand, indicating that increased fuel prices also lead to efficiency improvements associated with the shift from internal combustion engines to alternative drive train technologies. However, there is clear variation in the size of energy reduction on the one hand, and fuel substitution effects on the other across the models. MESSAGE, REMIND and WITCH show higher fuel substitution rates (48-83\% of the oil change), while this is less the case in the more technology-rich models POLES, IMAGE and TIAM-UCL (2-34\%).

\subsection{Service demand and fuel consumption elasticities}

For the models that include details of passenger transport modes (IMAGE, POLES and TIAM-UCL), Table 3 shows the mean and standard deviation in modal service demand (expressed in passenger kilometres (pkm) or tonne kilometres (tkm)) and energy efficiency elasticities of the three oil \& natural gas price shock scenarios. This method gives insight into the underlying sectoral changes, for example changes in the kilometres travelled or in the fuel efficiency of each transport mode, which contribute to sectors' change in energy demand. At the same time, it provides the opportunity to compare model elasticities to empirical data, which are often reported at modal level. For the REMIND, WITCH and MESSAGE projections, the contribution of service demand and efficiency to energy demand elasticities have been specified for total transport, freight and passenger elasticities, as shown in Table 3.

The passenger service demand elasticity-the elasticity of the travelled passenger kilometres-in 2030 varies between -0.2 and -0.3 across all models and in 2060 between -0.1 and -0.5 . Freight service demand ranges from -0.1 to -0.5 in 2030 and from -0.1 to -1.3 in 2060. In the REMIND model, where there is no alternative for liquid fuel in freight transport, fuel-price shocks have a larger impact on transport prices than in passenger transport, resulting in higher elasticity values. In all models, but REMIND the transport service demand is not elastic to fuel prices (i.e. $<1$ ). WITCH service demand and POLES freight service demand projections are not related to energy prices, but are driven only by GDP and population, and changes in energy prices are reflected only in the choice of technology. Not capturing service demand price elasticity could lead to relatively downward bias for the overall energy demand elasticity. Indeed, of the six models, POLES and WITCH energy demand elasticity are on the low side of the spectrum. MESSAGE does not differentiate between passenger and freight transport demand, but relates total transport (useful) energy demand directly to economic and demographic drivers.

Fouquet has analysed the income and price elasticities of passenger transport demand between 1850 and 2010 in the United Kingdom, and shows that both elasticities have declined over time (Fouquet, 2012), from 3.1 and -1.5 to 0.8 and -0.6 , respectively. Price elasticities depend on income effects as well as substitution effects. When incomes rise, the share of fuel expenditure in total expenditure declines, leading to lower price sensitivity. Moreover, with higher incomes travel time is valued more, and fuel costs take up a relatively smaller share of the generalised cost of travel (in which money and time

\footnotetext{
5 In the following, cross-price market share semi-elasticities are referred to simply as cross-price elasticities for brevity

${ }^{6}$ If the total quantity $\sum_{j=1}^{I} Q_{j}$ does not change, the standard cross price elasticity $\eta_{\mathrm{i}, \mathrm{j}}$ can be obtained from this elasticity as $\eta_{\mathrm{i}, \mathrm{j}}=\eta_{\mathrm{MSi}, \mathrm{j}} / \overline{\mathrm{MS}_{\mathrm{i}}}$ where $\overline{\mathrm{MS}_{\mathrm{i}}}$ represents the average market share of fuel $I$ in both scenarios.

7 Alternative fuel is defined as all fuels other than oil.
} 

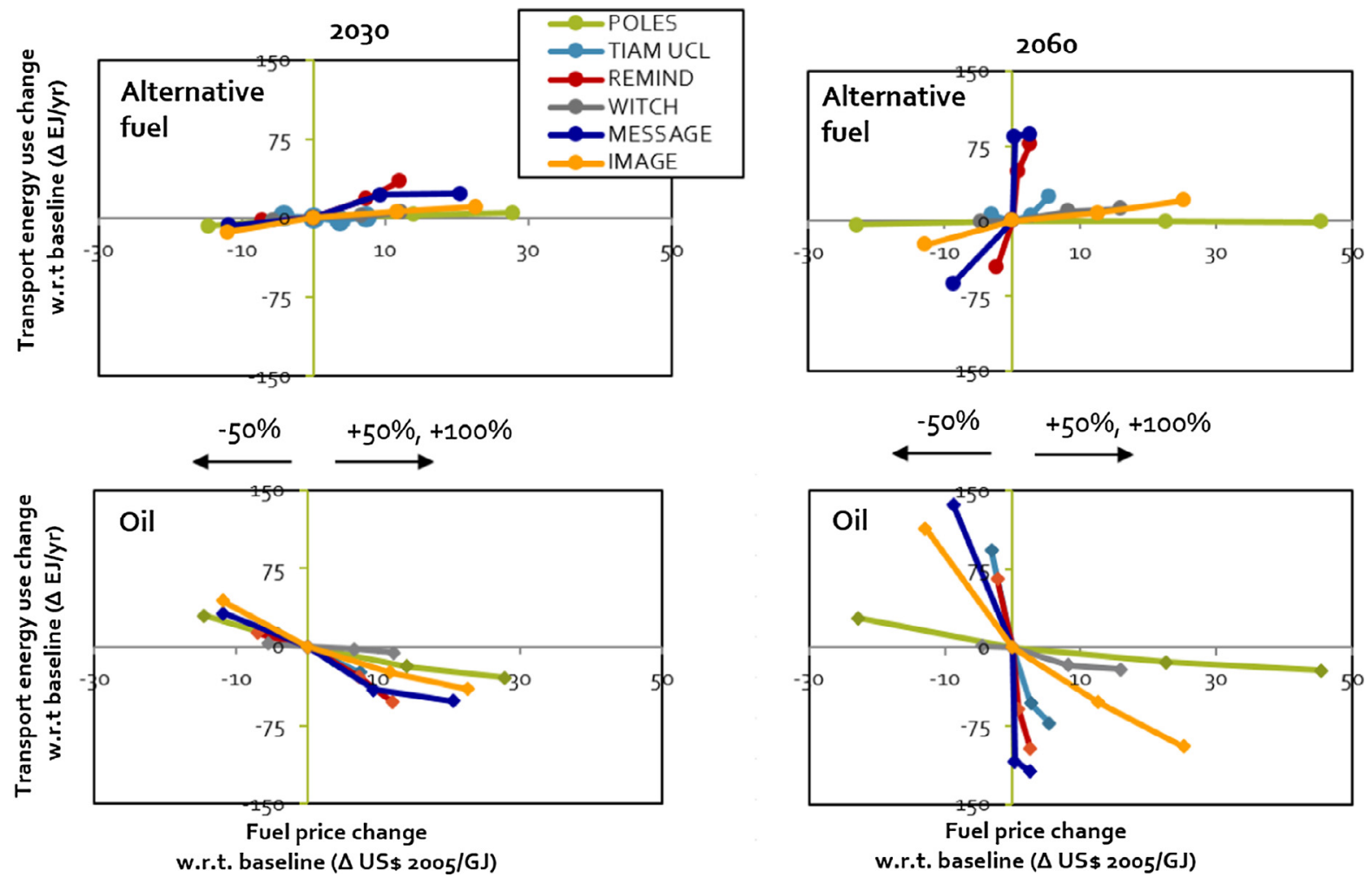

Fig. 3. The oil (bottom) and alternative fuel (AF) (top) energy demand response to oil and gas price shocks - Scenarios 2,5 and 8 - in 2030 (left) and 2060 (right). Alternative fuel is defined as any fuel other than oil.

Table 3

Mean service demand ( $\mathrm{kkm}$ or tkm), fuel efficiency (MJ/pkm or MJ/tkm) and fuel consumption (MJ) elasticities to oil price per mode of transport and aggregated for freight, passenger and total transport. Calculated by comparing the oil \& natural gas fuel-price shock Scenarios 2, 5 and 8 to the baseline. The standard deviation in the elasticity values of these three scenarios are indicated between brackets. In bold are the elasticities that are elastic $(>1)$.

\begin{tabular}{|c|c|c|c|c|c|c|c|}
\hline & & IMAGE & & POLES & & TIAM-UCL & \\
\hline & & 2030 & 2060 & 2030 & 2060 & 2030 & 2060 \\
\hline \multirow[t]{3}{*}{ LDV } & Pkm & $-0.2(0.1)$ & $-0.1(0.0)$ & $-0.2(0.0)$ & $-0.1(0.0)$ & $0.0(0.0)$ & $-0.1(0.0)$ \\
\hline & Efficiency & $-0.3(0.2)$ & $-0.7(0.6)$ & $-0.3(0.1)$ & $-0.3(0.0)$ & $-0.2(0.0)$ & $-2.0(0.7)$ \\
\hline & Energy & $-0.5(0.2)$ & $-0.8(0.6)$ & $-0.4(0.2)$ & $-0.4(0.0)$ & $-0.2(0.0)$ & $-2.1(0.7)$ \\
\hline \multirow[t]{3}{*}{ Public transport } & $\mathrm{Pkm}$ & $-0.2(0.0)$ & $-0.2(0.1)$ & $-0.2(0.0)$ & $-0.1(0.0)$ & $-0.1(0.0)$ & $-0.1(0.0)$ \\
\hline & Efficiency & $-0.1(0.2)$ & $-0.4(0.5)$ & $-0.3(0.0)$ & $-0.2(0.0)$ & $0.0(0.0)$ & $-0.4(0.2)$ \\
\hline & Energy & $-0.4(0.2)$ & $-0.6(0.4)$ & $-0.5(0.0)$ & $-0.4(0.1)$ & $0.0(0.0)$ & $-0.4(0.2)$ \\
\hline \multirow[t]{3}{*}{ Aviation } & Pkm & $-0.7(0.1)$ & $-0.6(0.1)$ & $0.1(0.0)$ & $0.0(0.0)$ & $-0.3(0.0)$ & $-0.4(0.1)$ \\
\hline & Efficiency & $-0.1(0.1)$ & $-0.6(0.2)$ & $-0.2(0.1)$ & $-0.2(0.1)$ & $0.0(0.0)$ & $0.0(0.0)$ \\
\hline & Energy & $-0.8(0.1)$ & $-1.2(0.1)$ & $-0.1(0.1)$ & $-0.1(0.1)$ & $-0.3(0.0)$ & $-0.5(0.1)$ \\
\hline Walking \& cycling & $\mathrm{Pkm}$ & $0.1(0.0)$ & $0.2(0.0)$ & & & & \\
\hline \multirow[t]{3}{*}{ Total passenger } & $\mathrm{Pkm}$ & $-0.2(0.0)$ & $-0.2(0.0)$ & $-0.2(0.0)$ & $-0.1(0.0)$ & $-0.2(0.0)$ & $-0.3(0.0)$ \\
\hline & Efficiency & $-0.3(0.1)$ & $-0.7(0.4)$ & $-0.2(0.1)$ & $-0.2(0.0)$ & $0.0(0.0)$ & $-\mathbf{1 . 0}(0.6)$ \\
\hline & Energy & $-0.5(0.2)$ & $-0.9(0.4)$ & $-0.4(0.1)$ & $-0.3(0.0)$ & $-0.2(0.0)$ & $-1.3(0.5)$ \\
\hline \multirow[t]{3}{*}{ Total freight } & Tkm & $-0.2(0.1)$ & $-0.1(0.1)$ & & & $-0.1(0.0)$ & $-0.1(0.0)$ \\
\hline & Efficiency & $-0.1(0.2)$ & $-0.3(0.3)$ & $-0.2(0.0)$ & $-0.1(0.0)$ & $-0.3(0.3)$ & $-2.0(1.9)$ \\
\hline & Energy & $-0.3(0.1)$ & $-0.4(0.2)$ & $-0.2(0.0)$ & $-0.1(0.0)$ & $-0.4(0.3)$ & $-2.1(1.9)$ \\
\hline \multirow[t]{3}{*}{ Total Transport } & Energy & $-0.4(0.1)$ & $-0.7(0.1)$ & $-0.3(0.1)$ & $-0.2(0.0)$ & $-0.3(0.1)$ & $-1.5(0.7)$ \\
\hline & & REMIND & & WITCH & & MESSAGE & \\
\hline & & 2030 & 2060 & 2030 & 2060 & 2030 & 2060 \\
\hline \multirow[t]{3}{*}{ Total passenger } & Pkm & $-0.3(0.1)$ & $-0.5(0.2)$ & & & & \\
\hline & Efficiency & $0.0(0.0)$ & $-1.7(0.7)$ & $0.0(0.0)$ & $-0.1(0.2)$ & & \\
\hline & Energy & $-0.3(0.1)$ & $-2.3(0.9)$ & $0.0(0.0)$ & $-0.1(0.2)$ & & \\
\hline \multirow[t]{3}{*}{ Total freight } & Tkm & $-0.5(0.1)$ & $-1.3(0.5)$ & & & & \\
\hline & Efficiency & $0.0(0.0)$ & $0.1(0.1)$ & $0.0(0.0)$ & $0.0(0.0)$ & & \\
\hline & Energy & $-0.5(0.1)$ & $-1.2(0.5)$ & $0.0(0.0)$ & $0.0(0.0)$ & & \\
\hline Total transport & Energy & $-0.3(0.1)$ & $-1.9(0.7)$ & $0.0(0.0)$ & $-0.1(0.1)$ & $-0.4(0.1)$ & $0.4(3.8)$ \\
\hline
\end{tabular}


are accounted for) (Small and Van Dender, 2007). Fouquet compares service demand to the price of service demand, instead of to the price of fuel (Fouquet, 2012). Therefore, the results in Table 3 cannot be compared directly to Fouquet's results. The described trends of service demand's reduced sensitivity to prices over time, can be seen for some modes of transport, but others show the opposite trend.

Most models show a response in efficiency change that is stronger for 2060-ranging from -0.1 to -1.7 for passenger transport and from -0.0 to -2.0 for freight transport-than for 2030. As a result, in all models except POLES, the longterm (2060) energy demand elasticity is higher than that in the medium term (2030), as is also noted in Section 3.1. This is especially pronounced in TIAM-UCL's projections. This is because (1) models have a much longer time period to respond to higher/lower prices, and (2) new vehicle technology developments have led to cheaper alternatives, which, for example in the case of electric vehicle deployment, would lead to higher efficiency. Also, long-term feedback effects on fuel prices, as seen in REMIND, MESSAGE and TIAM-UCL projections, could further enhance this effect.

A large share of the empirical research on transport price elasticity has focused on road transport elasticities to the petrol price under different circumstances, and a few review studies have summarised these results in 'generic values'. Johansson and Schipper (1997) study 12 OECD countries, for the period from 1973 to 1992, and find long term elasticities to fuel prices of car service demand to range between -0.05 and -0.55 , and of car fuel economy to range between -0.35 and -0.45 . These figures are comparable to those in reviews by Graham and Glaister (2002), Goodwin et al. (2004) and Espey (1998). Interestingly, the models' LDV service demand elasticities range from -0.1 to -0.2 , which is within that range. ${ }^{8}$ The models respond very similar, not covering the full uncertainty found empirically. For 2030, the efficiency response of the models ( -0.3 in all models) is very comparable to the empirically found data; leading to an overall comparable LDV energy consumption elasticity in 2030. For 2060 however, both IMAGE and TIAM-UCL project a stronger efficiency response, resulting in an elastic $(<-1)$ response that is beyond the range summarised in the reviews of empirically found elasticities. The availability of more fuel-efficient alternative types of vehicles increases the substitution effect on the price elasticity projected for the second half of the century.

The differences between price elasticities per transport mode, in the model projections, do not necessarily imply modal shifts, because the elasticity is defined as a relative decrease in $\mathrm{pkm}$ to the transport modes' total $\mathrm{pkm}$, and the transport modes differ in overall volume. Moreover, the various transport modes do not contribute equally to the overall transport volume (some, such as bicycles, have a smaller share). A change in fuel price can be expected to have a larger effect on the transport modes that are relatively high in energy consumption, such as LDVs and aviation. Fouquet argues that air transport is a 'luxury' form of transport and service demand would be more sensitive to fuel prices than would other modes of transport (Fouquet, 2012). IMAGE and TIAM-UCL indeed show higher service demand responses in aviation than in other modes of transport.

\subsection{Market share elasticities of fuel}

The transport sector is currently being dominated by oil products, but IAMs show that fuel switching is an effective way to mitigate the greenhouse gas emissions from the transport sector, in order to achieve a stringent climate target (Edelenbosch et al., 2017). The scenarios with oil, biofuel and electricity price shocks of $+100 \%$ (Scenarios 6,7 and 8 ) and $-50 \%$ (Scenarios 2, 3 and 4) are used to analyse how responsive fuel market shares are to fuel price changes for various carriers. Following the equations in Section 2.5, Fig. 4 shows the cross-price elasticities per fuel type. The interpretation, for instance for IMAGE, shows that a doubling ${ }^{9}$ of oil prices will lead to a 50 percentage points decrease in the market share of oil in transportation by 2050, whereas the share of biofuels and electricity will increase by respectively 18 and 21 percentage points.

Fuel market shares are considerably more responsive to oil and biofuel price changes than to electricity price changes. For many modes of transport, switching to electricity means switching to an alternative type of propulsion. The lower sensitivity could be explained by the fact that technology cost, availability and consumer behaviour are larger hurdles than the costs of electricity in relation to this transition. The fuel market is the most sensitive to changing oil prices and decreasing biofuel prices, which both lead to oil substitution. Elasticities of all fuel types in all models increase over time, with the exception of the IMAGE biofuel response in the $+100 \%$ scenario. POLES and WITCH show low response compared to the other models, projecting the sector to remain dependent on oil irrespective of fuel price changes, in line with the low responses to oil price changes as shown in Sections 3.1 and 3.2. MESSAGE and REMIND show a high response, which again partially can be explained by the feedback effects on prices, but also higher fuel switching flexibility due to less technology constraints.

Oil price increases are projected to lead to a switch from oil to biofuel in 2030 and, in some models to fossil synfuel, while in 2050 electricity also becomes an attractive alternative. Increasing electricity and biofuel prices lead to a reduction in the use of both fuel types, from which it can be concluded that, under the baseline scenario, electricity and biofuel have a certain share in transport fuel use. In REMIND, intertemporal foresight and interactions with other fuel-consuming sectors may lead to the opposite effect; for example, showing increasing oil shares in reaction to higher oil prices in 2030. Lowering fuel prices

\footnotetext{
${ }^{8}$ Note that the IAM values are expressed in passenger kilometres (pkm) and thus car sharing effects and load factor change are accounted for in energy intensity change, which could explain the somewhat low values.

${ }^{9}$ Scenarios (8-10) were precisely designed to implement a doubling of fuel prices, compared to those in the baseline. In the cross elasticity calculation, the price increase is calculated relative to the average price shock and the baseline scenario $\left(\Delta \mathrm{P}_{\mathrm{j}}\right) /\left(0.5\left(\mathrm{P}_{(\mathrm{j}, 1)}+\mathrm{P}_{(\mathrm{j}, 2)}\right)\right.$. Therefore, the relative price increase is $+2 / 3$.
} 

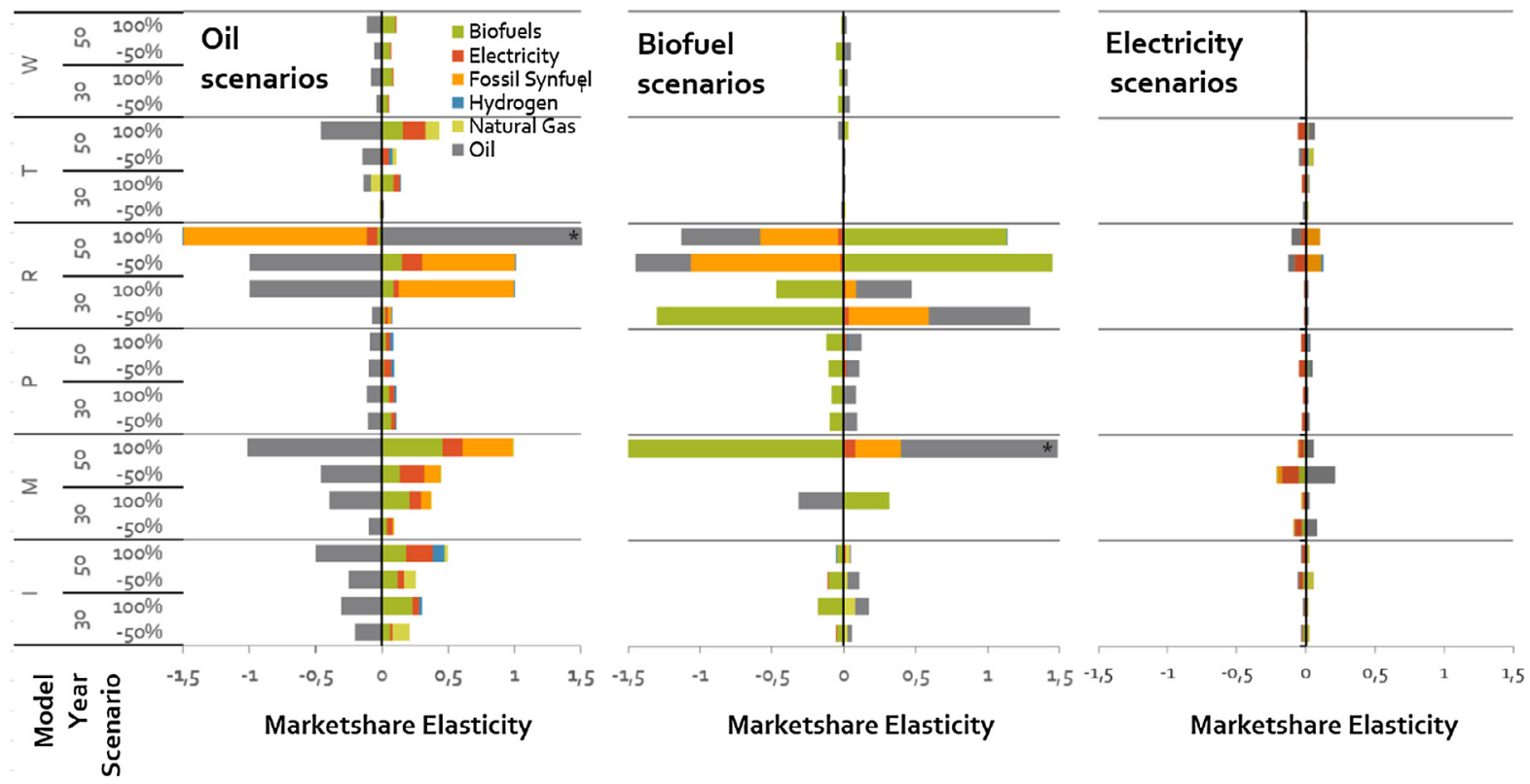

Fig. 4. Market share elasticities in response to changes in oil, biofuel and electricity prices from $+100 \%$ to $-50 \%$. Elasticities indicate the shift in market shares, for all the different fuel types for which the sum of the elasticities is 0 . Negative elasticities in the $-50 \%$ scenario imply an increase in use, as the elasticity is relative to the price signal. The models are indicated by their first letter $(R=$ REMIND, $I=I M A G E, T=T I A M-U C L, M=M E S S A G E, P=P O L E S$ and $\mathrm{W}=\mathrm{WITCH}$ ). ${ }^{*}$ In two scenarios (marked with ${ }^{*}$ ) the market elasticity was larger than 1.5 - due to very high price feedbacks- the results were normalised to 1.5 .

leads to strong early consumption, both in the transport sector and in other sectors, which implies that long-term scarcities become more pronounced, in turn leading to increased long-term reliance on alternative fuels.

\subsection{Income elasticities}

To assess the sensitivity of demand to income level, an approach similar to that described in Section 3.1 was used, distinguishing the effects of changes in efficiency and service demand. The results are presented in Table 4 . There have been suggestions that at higher income the per capita kilometres travelled would saturate and that we are reaching peak travel (Millard-Ball and Schipper, 2011; Dargay et al., 2007). The theory is that transport is perceived as a 'luxury' product which is sensitive to income changes. As incomes continue to rise, saturation effects will reduce the income elasticity. This theory is supported by the already mentioned reduced travel demand to income elasticity between 1850 and 2010, shown by Fouquet (2012). This trend is not clearly reflected in all the model results, neither over time nor with increased income. The elasticity values for high and low incomes are rather comparable, although IMAGE, REMIND and WITCH do show lower service demand elasticities to higher income scenarios.

The energy efficiency response in IMAGE increases, due to a shift to higher intensity transport modes with higher income. In the other models, efficiency decreases, which could reflect the concept of a larger budget leading to an increase in technology use, which, in turn, leads to efficiency learning. Johansson and Schipper (1997) find that the long-term elasticity of vehicle fuel consumption (related to what here is called efficiency) with respect to income is between 0.05 and 1.6, and Graham and Glaister (2002) report this to be between 1.1 and 1.3. These two studies were conducted at LDV level, and, therefore, are not easy to compare to the model projections used in this study, as the effects of structural changes (shifting transport modes) are not included, but it can be concluded that this positive relationship is not necessarily reflected in the models. Both studies also analyse long-term elasticity of service demand with respect to income; Johansson and Schipper (1997) find a range of 0.65-1.25, while Graham and Glaister (2002) report this to be between 1.1 and 1.8. For the United Kingdom, Fouquet reports a reduction in transport service demand to income elasticity of 3.1-1.0 (including air travel) between 1850 and 2010. Compared to these figures, the IAM service demand elasticity values are on the low side, with the exception of the WITCH model. Income elasticities of transport energy demand are reported to be greater than price elasticities provided in the literature (Goodwin et al., 2004). The models show service demand to income elasticities are indeed larger (especially in WITCH). Negative energy efficiency elasticities lead to income energy demand elasticities (ranging from 0.31 to 1.44 ) that are comparable to price elasticities. 
Table 4

Service demand (pkm or tkm), fuel efficiency (MJ/pkm or MJ/tkm) and fuel consumption (MJ) elasticities with respect to income changes (Scenarios 11 and 12 ) for freight, passenger and total transport.

\begin{tabular}{|c|c|c|c|c|c|c|c|c|c|c|}
\hline & \multicolumn{2}{|c|}{ IMAGE } & \multicolumn{2}{|c|}{ POLES } & \multicolumn{2}{|c|}{ REMIND } & \multicolumn{2}{|c|}{ WITCH } & \multicolumn{2}{|c|}{ TIAM-UCL } \\
\hline & low & high & low & high & low & high & low & high & low & high \\
\hline \multicolumn{11}{|c|}{ Passenger transport in 2030} \\
\hline Pkm & 0.50 & 0.38 & 0.49 & 0.65 & 0.45 & 0.32 & 1.19 & 0.91 & & \\
\hline Efficiency & 0.11 & 0.20 & -0.13 & -0.17 & -0.01 & -0.02 & -0.03 & -0.10 & & \\
\hline Energy & 0.61 & 0.58 & 0.36 & 0.47 & 0.44 & 0.31 & 1.15 & 0.81 & & \\
\hline \multicolumn{11}{|c|}{ Freight transport in 2030} \\
\hline $\mathrm{Tkm}$ & 0.87 & 0.35 & 0.43 & 0.83 & 0.42 & 0.30 & 1.17 & 0.93 & & \\
\hline Efficiency & -0.26 & 0.18 & -0.01 & -0.42 & -0.06 & 0.00 & -0.03 & 0.04 & & \\
\hline Energy & 0.61 & 0.54 & 0.42 & 0.41 & 0.36 & 0.31 & 1.14 & 0.97 & & \\
\hline Total & 0.61 & 0.56 & 0.39 & 0.44 & 0.41 & 0.31 & 1.15 & 0.87 & 0.65 & 0.99 \\
\hline \multicolumn{11}{|c|}{ Passenger transport in 2060} \\
\hline Pkm & 0.51 & 0.53 & 0.62 & 0.40 & 0.37 & 0.31 & 0.96 & 0.75 & & \\
\hline Efficiency & 0.16 & 0.38 & -0.09 & -0.18 & 0.08 & 0.04 & -0.06 & -0.30 & & \\
\hline Energy & 0.67 & 0.90 & 0.52 & 0.22 & 0.45 & 0.34 & 0.91 & 0.46 & & \\
\hline \multicolumn{11}{|c|}{ Freight transport in 2060} \\
\hline Tkm & 0.78 & 0.50 & 0.53 & 0.59 & 0.41 & 0.25 & 0.99 & 0.79 & & \\
\hline Efficiency & -0.08 & 0.03 & -0.14 & -0.15 & -0.02 & 0.00 & 0.02 & 0.04 & & \\
\hline Energy & 0.70 & 0.52 & 0.40 & 0.44 & 0.39 & 0.25 & 1.01 & 0.83 & & \\
\hline Total & 0.68 & 0.77 & 0.47 & 0.32 & 0.43 & 0.31 & 0.95 & 0.62 & 0.53 & 1.44 \\
\hline
\end{tabular}

\section{Discussion and conclusions}

In this paper, we introduced fuel price shocks in IAMs in order to determine the implicit transport demand elasticities. This can help to describe and understand model behaviour and projected results. In the experiment set-up, ideally, the fuel price shocks would follow the exact same fuel price pathways in all models. However, the fuel price trajectories in the baseline already varies across models. Moreover, due to interference with some of the models solution methods, fuel prices could not follow a predefined pathway in all models. In those models price increases/decreases were added to endogenously calculated fuel prices to mimic the fuel price shocks. In some models, this method resulted in fuel prices moving away from the set pathway over time, as a result of for example lower fuel use. In REMIND, fuel prices also moved away but already in the early decades due to intertemporal foresight. Because of the relative nature of elasticities, different fuel price pathways not necessarily have an impact on the results, but we did find the demand response to be both pathway- and time-dependent. This is most clearly demonstrated by the results from the MESSAGE and REMIND models, projecting large demand differences, while fuel price differences became very small (in some cases, even negative) by 2060 . Remaining as close as possible to the intended fuel price pathway would therefore improve the comparability of results between models. However the scenarios do show how the different solution methods affect the model dynamics. It can be expected that the implementation of a carbon tax could result in similar model responses.

On the basis of the results, the following conclusions can be derived:

The proposed method in this paper to derive price and income elasticities as diagnostic indicators provides a transparent environment to test model dynamics. The approach provides insights into model responsiveness, both for the medium and long term. It enables us to evaluate model behaviour and to distinguish a model's fingerprint. At the same time, it could be used to understand the effect of model development on model behaviour, through a before-and-after comparison. Modelling individual transport modes explicitly does not lead to apparent differences in energy demand responses (compared to models that only represent transport modes in a more aggregated way). The detailed and less detailed models show similar elasticity values.

Efficiency and service demand elasticities to fuel price are within the range of values found empirically, and very close to each other in the medium term. Comparing model elasticities at the modal level, and specifying between service demand and efficiency changes, shows that in 2030 energy demand elasticities are very comparable between models and close to the range reported in the literature. This shows that in terms of historical validation in the medium term the models perform well. LDV energy demand elasticities to oil and gas prices are projected to range from -0.2 to -0.5 in 2030 . Total transport energy elasticity values, projected to range between 0.0 and -0.4 in 2030 , are also comparable (although on the low side) to the values of a similar experiment reported by Hogan and Sweeney (Hogan and Sweeney, 1981).

A division can be made between the models that become more responsive in the long term (2060) than in the medium term (2030). Some models clearly show higher fuel switching and energy demand reduction responses in the long term, while service demand response remains comparable. The projected elasticity of total energy demand in transport to oil and natural gas prices in 2060 range from 0.4 to -1.9 , and for LDV energy consumption from -0.4 to -2.1 . There are however different effects that can have caused the increased response. In IMAGE, REMIND, MESSAGE and TIAM-UCL, alternative technologies become more attractive (cheaper) in the long term, and therefore oil price changes can lead to a stronger response. 
In REMIND's freight sector, the opposite is visible since no alternatives are available. Therefore, if oil prices increase freight transport becomes more expensive and, thus, leads to higher price effects on service demand. MESSAGE, REMIND and TIAMUCL also show large feedback effects on fuel price pathways in the long term, while demand does not immediately follow. This shows that near term price policies could have long term effects. Assuming service demand pathways exogenously, as is done in WITCH and POLES, leads to a relatively weak demand response.

Market share distribution responds more strongly to oil and biofuel price changes than to electricity prices. Oil will be substituted as the dominant fuel when oil prices increase. Biofuel price change sees in some models a strong effect but electricity price changes hardly have an impact on the projected shares. The models show that, in 2030, mainly biofuel is used as a substitute, and some models use fossil synfuel, while electricity shares increase as a result of higher oil prices in the long term. Furthermore, the models show a stronger response to biofuel price reductions than to reductions in the oil price. The models are not responsive to electricity price changes, indicating that other factors such as technology costs and consumer behaviour might be more important in this transition. The models' response to a price jump of $50 \%$ compared to a price jump of $100 \%$ is not clearly different. Elasticity values for most models are comparable under both these scenarios, implying a linear demand response. Again, a clear difference can be seen between models that show a high response (REMIND, MESSAGE), medium response (IMAGE, TIAM-UCL) and a low response (WITCH and POLES).

Service demand projections are more responsive to income level than to fuel prices, which corresponds to empirical findings. The model results are responsive to income projections and elasticity values range between 0.31 and 1.44 . This is within the range reported in the literature. Even so, this range has a large impact on the projected transport demand, and could explain the varying transport sector service demand growth projections which have been seen in previous model comparison studies (Edelenbosch et al., 2017). Reduced income elasticities over time, or in response to higher income shocks indicating saturation, cannot clearly be retraced in the model results. A better understanding of the uncertainty of income effects on service demand by exploring different income pathways as well as different service demand to income elasticities, is very relevant-as is having a better understanding of the role of saturation. The efficiency response to income change differs across models. In some models efficiency increases as a result of technology learning, while in others it decreases due to a shift to more energy-intensive transport modes.

\section{Acknowledgements}

The research leading to these results has received funding from the European Union's Seventh Framework Programme FP7/2007-2013, under grant agreement no. 308329 (ADVANCE).

\section{Appendix A. Supplementary material}

Supplementary data associated with this article can be found, in the online version, at http://dx.doi.org/10.1016/j.trd. 2017.03.005.

\section{References}

Bucklin, R.E., Russell, G.J., Srinivasan, V., 1998. A relationship between market share elasticities and brand switching probabilities. J. Mark. Res., 99-113 Carrara, S., Longden, T., 2017. Freight futures: the potential impact of road freight on climate policy. Transp. Res. Part D: Transp. Environ. 55, 359-372. http://dx.doi.org/10.1016/j.trd.2016.10.007.

Clarke, L. et al, 2009. International climate policy architectures: overview of the EMF 22 international scenarios. Energy Econ. 31 (Supplement 2 ), S64-S81. Dargay, J., Gately, D., 1997. The demand for transportation fuels: imperfect price-reversibility?. Transport. Res. Part B: Methodol. 31 (1), 71-82.

Dargay, J., Gately, D., Sommer, M., 2007. Vehicle ownership and income growth, worldwide: 1960-2030. Energy J., 143-170.

Edelenbosch, O., McCollum, D., et al, 2017. Decomposing passenger transport futures: comparing results of global integrated assessment models. Transport. Res. Part D: Transp. Environ. 55, 281-293. http://dx.doi.org/10.1016/j.trd.2016.07.003.

Espey, M., 1998. Gasoline demand revisited: an international meta-analysis of elasticities. Energy Econ. 20 (3), $273-295$.

Fouquet, R., 2012. Trends in income and price elasticities of transport demand (1850-2010). Energy Policy 50, 62-71.

Girod, B. et al, 2013. Climate impact of transportation A model comparison. Climatic Change 118 (3-4), 595-608.

Goodwin, P., Dargay, J., Hanly, M., 2004. Elasticities of road traffic and fuel consumption with respect to price and income: a review. Transp. Rev. 24 (3), 275292.

Graham, D.J., Glaister, S., 2002. The demand for automobile fuel: a survey of elasticities. J. Transp. Econ. Pol., 1-25

Hogan, W., Sweeney, J., 1981. Aggregate elasticity of energy demand. Energy J. 2 (2), 37-75.

Jaccard, M., Beylin-Stern, A., 2014. Hybrid Simulation Modeling to Estimate U.S. Energy Elasticity of Substitution. Simon Fraser University.

Johansson, O., Schipper, L., 1997. Measuring the long-run fuel demand of cars: separate estimations of vehicle stock, mean fuel intensity, and mean annual driving distance. J. Transp. Econ. Pol., 277-292.

Karkatsoulis, P., Siskos, P., et al, 2017. Simulating deep $\mathrm{CO}_{2}$ emission reduction in transport in a general equilibrium framework: the GEM-E3T model. Transport. Res. Part D: Transp. Environ. 55, 343-358. http://dx.doi.org/10.1016/j.trd.2016.11.026.

Kriegler, E. et al, 2015. Diagnostic indicators for integrated assessment models of climate policy. Technol. Forecast. Soc. Chang. 90, 45-61.

Litman, T., 2013. Understanding transport demands and elasticities. How prices and other factors affect travel behavior (Victoria Transport Policy Institute: Litman) Available at <http://www.vtpi.org/elasticities.pdf> [Verified 22 November 2013].

Luderer, G. et al, 2012. The economics of decarbonizing the energy system-results and insights from the RECIPE model intercomparison. Clim. Change 114 (1), 9-37.

McCollom, B.E., Pratt, R.H., 2004. Traveler response to transportation system changes. Chapter 12 - Transit Pricing and Fares.

McCollum, D. et al, 2014. Transport electrification: a key element for energy system transformation and climate stabilization. Clim. Change 123 (3-4), 651664.

McCollum, D.L., Wilson, C., et al, 2017. Improving the behavioral realism of global integrated assessment models: an application to consumers' vehicle choices. Transport. Res. Part D: Transp. Environ. 55, 322-342. http://dx.doi.org/10.1016/j.trd.2016.04.003. 
Millard-Ball, A., Schipper, L., 2011. Are we reaching peak travel? Trends in passenger transport in eight industrialized countries. Transp. Rev. 31 (3), $357-$ 378.

Mittal, S.H., Dai, H., et al, 2017. Key factors influencing the global passenger transport dynamics using the AIM/Transport model. Transport. Res. Part D: Transp. Environ. 55, 373-388. http://dx.doi.org/10.1016/j.trd.2016.10.006.

Ó Broin, E., Guivarch, C., 2017. Transport infrastructure costs in low-carbon pathways. Transport. Res. Part D: Transp. Environ. 55, 389-403. http://dx.doi. org/10.1016/j.trd.2016.11.002.

O'Neill, B.C. et al, 2014. A new scenario framework for climate change research: the concept of shared socioeconomic pathways. Clim. Change 122 (3), $387-$ 400 .

Pietzcker, R.C. et al, 2014. Long-term transport energy demand and climate policy: alternative visions on transport decarbonization in energy-economy models. Energy 64, 95-108.

Riahi, K., et al., 2012. Chapter 17 - Energy Pathways for Sustainable Development. In: Global Energy Assessment - Toward a Sustainable Future: Cambridge University Press, Cambridge, UK and New York, NY, USA and the International Institute for Applied Systems Analysis, Laxenburg, Austria. p. $1203-1306$. Small, K.A., Van Dender, K., 2007. Long run trends in transport demand, fuel price elasticities and implications of the oil outlook for transport policy.

Sugiyama, M. et al, 2014. Energy efficiency potentials for global climate change mitigation. Clim. Change 123 (3-4), $397-411$.

Ziemba, W.T., Schwartz, S.L., 2012. Energy Policy Modeling: United States and Canadian Experiences. Specialized Energy Policy Models, Vol. I. Springer Science \& Business Media. 